International Journal of Scholarly Papers for Media and Society Research

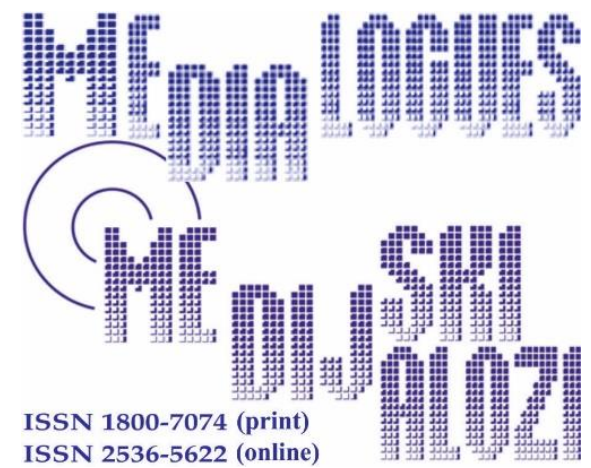

Stamenkovic, M. (2012), „In Full View - Cybersuicide, Hypervisual Self and Public Showcase of Death (For Real)", Media Dialogues / Medijski dijalozi,

Vol. 14, No. 2, pp. 39-62.

\title{
In Full View - Cybersuicide, Hypervisual Self and Public Showcase of Death (For Real)
}

\author{
MARKO STAMENKOVIC, Researcher, Doctoral Student \\ University of Ghent, Department of Philosophy and Moral Sciences, Center \\ for Ethics and Value Inquiry \\ Ghent - Belgium
}

\begin{tabular}{|c|c|c|}
\hline $\boldsymbol{A} \boldsymbol{R} \boldsymbol{T} \boldsymbol{I} \boldsymbol{C L} \boldsymbol{E}$ & Received: December 11,2021 / & Revised from: January 12, 2021 \\
$\boldsymbol{I} \boldsymbol{N} \boldsymbol{F} \boldsymbol{O}$ & Accepted: December 12,2021 / & Available online: April 15, 2021 \\
\hline $\boldsymbol{D O I}$ & doi.org/10.14254/1800-7074/14-2/3 \\
\hline
\end{tabular}

\begin{abstract}
The practice of suicide has taken on a new meaning in the last decade: alongside the advent of media technologies and cybernetic communication systems, it has become an unbridled pursuit of global visibility. The transnational character of such an increasingly extended and accelerated visibility resides exactly in those possibilities of computer technologies that have become available through digital images
\end{abstract}


and the role played by vision. By putting into focus the hyper-visual properties of the Internet, as an aspect easily overlooked by medical studies around the troubling and increasing phenomenon of cybersuicide, I am arguing for a more nuanced, inclusive and interdisciplinary approach, complementary to the existent and valuable body of work already achieved by the scientific research in the context of medicine and psychiatry. This paper therefore treats the contemporary culture of display in general and the overexposure of self-inflicted death through the virtual social environment in particular as interdependent and highly mobile kinds of cultural practice: it highlights the nature of cybersuicide as a real-time event and a spectacle over the channels of popular social networks that have facilitated not only its live recording and transmission over the Internet, but also its online documentation, archiving and the possibility of repetition (in an al-most ritualistic way) for massive voyeurism at any given moment in time by any cyber-spectator. In order to get closer to the complexity of the term itself and to the individual and group responsibility it puts forward, I am dealing with cybersuicide as "Internet-assisted" suicide and the relevance of the Internet for understanding the ideation of self-inflicted death: not only as pertaining to the textual or hyper-textual self (embodied by verbal online communication) but also to the visual or hypervisual self, the kind of "self" that uses a new site for the public showcase of its own real death in full view - precisely the virtual domain of the Internet. For a human being thus exposed, is it assistance (in the process of looking) that becomes a mode of survival, of overcoming death, a "liberation" from it - as opposed to non-assistance which leads to the condemnation of death?.

KEYWORDS: Suicide, cybersuicide, hypervisual self, public display of (selfinflicted) death, webcam, communications.

\section{INTRODUCTION}

One of the major concerns in the current psychiatric debates around suicide - as applied to the media in general and the Internet in particular is related to the role that the computer-generated systems, services and devices play in suicide prevention and, unfortunately, also in suicide stimulation. Some of the leading scholars in the field of suicide studies, from Europe and beyond, have questioned in a number of texts the peculiar relationship between the computer technology and suicide throughout the last decade, when the popular spread of Internet communication has become prominent as a worldwide phenomenon, now reaching the number of more than 2.2 billion users (Internet World Stats, 2012).

The potential influence of the Internet on its suicide-sensitive users was examined and put into question by the researchers in the field as early as 1990s (Baume et al., 1997). This was especially relevant as regards the influence exerted upon the young people (teenagers and adolescents). Firstly, it relates to their higher level of vulnerability in comparison to the elderly parts of the population. Secondly, it also 
relates to the inevitable, all the more increasing dependence - of their cultural habits and the personal, social and emotional domains of their everyday life - on the participation in the cyber communication (Wakefield and Rice, 2008). These are only two reasons why the issue at hand addresses the youth in particular. This kind of continuous interdependence has shown in many cases the high growth rate toward the addiction attitudes among the youth, due to their very frequent and long daily exposure to the Internet resources, which also includes their easy and free access to the suicide-related data available online. Accordingly, while introducing the term "cybersuicide" into the scientific literature, the early stages of research have been primarily concentrated on the effect of the existent interactive suicide notes on the behavior of young Internet users (Baume ee al., 1997).

This initial research approach has been developed throughout the last fifteen years into a trend showing an increasing interest in the verbal nature of suicideoriented communication over the Internet (especially when it comes to the analysis of its negative effects). The methodology applied would usually conceive of a series of steps, mostly relying upon the verbal/textual characteristics of analysis: scanning of webpages through selected keywords of scientific relevance (main identifier combined with co-identifiers, for example: "suicide" with "prevention" or "note") via scholar or ordinary digital search engines and directories; textual database analysis following the relevant patterns preconceived upon the traditional material resources and methods (print publications, for example); focus on verbal descriptions of suicide methods and communication in pro-suicide websites, as well as on textual characteristics of messages written and exchanged in suicide notes, etc. The formalization and institutionalization of research analyses thus achieved (in accordance with the predominance of verbal over any other type of exchange) has been valuable in reaching the goals toward suicide prevention. However, it has also showed certain limits by its general reliance upon the verbal nature of human communication, thus establishing a formal and even exclusive procedure in the official analysis of the pathological Internet use nowadays.

Being relatively "novel" among the phenomena under the scrutiny of a medical gaze, the pathological Internet use in general and the so-called cybersuicide in particular have been investigated by methods that rely on the Western logic of the "civilized" gaze of the worldwide population, namely - the logic of the word. By privileging speech over images, the visual character of digital communication technologies has been left aside in most of the studies related to cybersuicide. Nonetheless, throughout the not so long history of the Internet as the HyperText Markup Language (HTML, initiated in the late 1950s and early 1960s in the United States in the symbiotic context of scientific and military purposes) the visuality has gradually developed into a privileged domain of the global network cultures as we experience it today. The introduction of the visual element to the coding language in the early 1990s is a simple yet very significant fact that should be taken more seriously into account in the study of cybersuicide. 
Once the World Wide Web became configured to display graphic material, beside the display of hypertext documents, a new domain of communication possibilities opened up: "The added element of the visual, along with the ease and freedom of electronic movement enabled by hypertext, pushed what was an obscure resource available to engineers and technocrats into the churning mainstream of popular culture and global commerce" (Ferranto, 2010). One could even say that the existent body of work concerning the troubling issue of cybersuicide - insisting on the verbal features of online communication - has so far been generated by relying almost exclusively upon the hypertextual property of the Internet. The hypertextuality is, however, only one of its main characteristics: by introducing the graphic component, the hypervisual property of the Internet has gained the kind of prevalence that has not only become all the more evident nowadays but plays the most essential role in the global digital communication. Accordingly, the contemporary culture of display has become so much intertwined with the structural and functional properties of the digital communication technologies embodied by the Internet that cybersuicide presents but one of the components that have inevitably become embedded in those properties. It is precisely from this point of view that I want to address the notion of cyber-suicide in this paper alongside its implications both inside and outside of the virtual social environment.

The exclusion of hypervisibility from the scientific research of cyber-suicide shows only one side of the spectrum. On the other side, the clinical setting of neuroscience has been concerned with a relatively novel use of techniques and methods dealing with the procedures of the brain structure and functioning through the socalled neuroimages. As a visual scientific method of mapping the information generated in or by the brain and stored in its "database", neuroimaging remains a privileged domain of neuroradiologists. Even if the neuroimaging is associated with the suicide research (which is, actually, the case) it gives but one possible way of addressing the significance of visuality and image-oriented research. In the context of suicide, and cybersuicide in particular, how many people (including the suicide-sensitive Internet users) are aware of the "virtual" reality constructed by the neuroimages in the brain, and who of them would know what such a reality actually means? Not too many, I believe. Perhaps only a few of them, or nobody.

This brings us back to the very beginning of questioning the roles played by images in the context of cybersuicide, especially the status of (hyper) visuality and the functioning of gaze within the suicide-related (verbal and visual) communication over the Internet. The aim of this paper is not to give immediate answers to the questions that must be posed in this context: a further analysis revolving specifically around these issues shall be enforced among scholars of various disciplines. My modest con- tribution will be limited by some early and personal ideas that are proposed within the body of this paper, as a rough draft rather than an in-depth analysis. If conditions for such an in-depth analysis would be beneficial enough, a more profound investigation shall be developed and finalized in the forthcoming paper thro- 
ughout this year (2012), focused specifically on what I would like to name " $a$ suicidal gaze."

For the paper at hand, I would prefer to remain within the limits of its format, and will only accentuate the borderlines of the coordinate system of thought that brought me into thinking about the question "What is suicidal about vision?" My main concern here will be cybersuicide: the complexity of the notion itself, the individual and group responsibility it puts forward, and its relevance for understanding the ideation of self-inflicted death not only as pertaining to the textual or hypertextual self (embodied by verbal communication over the Internet) but also to the visual or hypervisual self. The notion of hypervisual self, as I understand it, denotes the kind of self that-in order to make visible (communicate, announce, stage, perform, document and confirm) its own suffering - uses a new site for the public showcase of its own real death, in full view: the virtual domain of the Internet. As mentioned earlier, already in the 1990s a new term has been coined in order to describe and define the particular nature of the evident inter-twining relationship between the role played by the World Wide Web as a tool for global informati- on exchange and the emerging trend of self-destructive attitudes among the youth.

"Cybersuicide" has thus become a common deno-minator for a phenomenon that has gained more and more prominence throughout the 2000s. However, the interpretation of the term has not been fixed to a single meaning: it can vary both among the specialists and the laymen. In order to start any future discussion concerning the issue I find it necessary to outline, from a personal perspective, an exemplary case alongside the terminological landscape within which a variety of meanings stem in regard to the notion of cybersuicide as it has been approached and understood in the recent scientific debates, as well as in the critical media theory and popular online communication.

\section{CYBERSUICIDE: A LONG SPECULATIVE HISTORY OF THE TERM}

The etymology of the term "suicide" alone stems from the Latin verb sui caedere, which translates into English as "to kill oneself". It denotes an act pertaining to, or leading to, self-destructive, ruinous act, fatal to one's life: a personal act by which one's own death is intentionally caused. What could we say about a specific kind of suicide if we would add a prefix "cyber" thus forming a new word ("cybersuicide")? In its antique Greek origins, $\kappa v \beta \varepsilon \rho$ (Eng. governmental) denotes a relation to the notion of governance, order, (navigation) control. Widely used and recognized as an Internet- related prefix (similar to other terms in the same chain of signifiers, such as "virtual", for example), all things "cyber", we could say today, relate to those contemporary phenomena that are based on the proliferating use of "high tech" information technologies, dependence on computer-networks and their respective operative systems, as well as on the Internet access thus facilitated (cybernetics, cyber- 
space, cybercafé, cyberpunk, even cybersex). The sense of controlling power inherent to the original Greek term is significant: it reveals an etymological and historical precondition for the humankind of our times, in a way that their inevitable dependence upon the cyberworld shall be understood as the sign of global governance by a new Internet regime to which they are all subjected.

Most of the recent theories facing the phenomenon of cybersuicide have come from the medical and psychiatric fields. They have stressed the link between the ideations of suicide on the Internet and the websites that not only contain information about "various methods and approaches to deliberate self-harm" but also provide easy access to such kind of information. This is all coupled with the high "rate at which information is dispersed" so that it could "contribute to the promotion of "offing' one's self which is particularly appealing to adolescents" (Birbal et al., 2009). The threat of a possible risk-increasing influence of the Internet has been repeated continuously in scientific efforts to bring its pathological use closer to our attention (Durkee et al., 2011).

This particularly concerns the pathological Internet use in relation to suicidality, namely "those cases of suicide and suicide attempts in which a direct connection to the Internet can be demonstrated [through] different applications of the Internet, such as reading online newspapers, use of Internet Relay Chat (IRC) and consulting Web pages with pro-suicide messages," all of them carrying a potential threat in exerting an effect on the suicidal behavior (D'Hulster and van Heeringen, 2006; Alao $\mathrm{AO}$ et al., 2006). Besides, following the results of some recent studies, we could also argue that since Internet has become an integral part of the life of millions of people in the developed and the developing world, it comes as no surprise that it has also, and unfortunately, become an integral part of their death (Sher and Vilens, 2009). By the risk it contains, it has certainly become an integral part of the potentiality of life-threat.

In their article "The Role of the Internet in suicide prevention" (2009) Swedish scientists Michael Westerlund and Danuta Wasserman use the term net suicides in order to analyze and frame the forms of online pro-suicide communication as opposed to the forms of online communication that defend a suicide preventive view. By contrasting the traditional media ( $\mathrm{TV}$, radio, press) to the medium of Internet, they are stressing the need not to "underestimate the role played by the Internet in shaping attitudes towards suicide among the general public" (Westerlund and Wasserman, 2009). The implications, both positive and negative, instituted by these forms of communication on actual human beings address their latent attitudes turning real upon consulting the web-based online contents. We shall accordingly challenge those impli- cations, first of all, by being able to transgress the divisions between two types of social environments to which the generation of our times is nece-ssarily subjected - the virtual ("real time") and the actual ("real life") social environment as they function in a rather complementary and mutually dependent manner. This is an important point to be taken more seriously into account, since the virtual social 
settings possess and offer an open access both to the positive and constructive as well as the negative and destructive elements inherent to those environments; once those elements transla- te into the opportunities and even values of significance for the Internet users, the consequences become more and more serious than we could have expected (in the negative case - even very dangerous and life-threatening): The Internet has thus provided a previously non-existent opportunity to publish material and discuss, confess and seek contact on a subject that has always been strongly taboo and therefore 'belonged' to only a few voices in public discourse. This opportunity has resulted in both constructive and strongly destructive contributions. (Westerlund and Wasserman, 2009).

From a more "popular" perspective, some other types of definitions are also available - where else but on the Internet itself. I am aware of the fact that the meaning of the words in dictionaries proposed by online encyclopedia (such as the Wikipedia, for example) imply a high risk of arbitrary interpretations that are usually not considered a reliable source of information, especially when it comes to a serious research. Nonetheless, I would like to indicate one of the possible formulations of the term "cybersuicide" exactly as it is presented by the Wikipedia, for a couple of very simple reasons:

- its popularity and frequency of consulting among the general population;

- its application of the term "assistance": by referring to cybersuicide as a form of suicide "assisted by computer technology" it gives an orientation that could be of significance for further examination toward what I would like to name "Internet-assisted" suicide;

- its application of the visual communication machines (Webcam) that facilitate the public display of suicidal behavior in "full view" in the online context.

In most of the Index sections of the available literature on cybersuicide the term "Internet-assisted suicide" as such does not appear. Its use is either very rare or extremely limited, in the sense that it offers an explanation of those kinds of suicides that have been facilitated, in one way or another, through online communication between the victim and someone else. The terms of significance (as related to "the Internet" and/or "assisted suicide" as the main identifiers) would include, among others: "legislation and regulation", "online therapy", "pro-suicide content", "selfhelp interventions", "suicide-pacts", "suicide prevention", "suicide-related material", "Netherlands", "Oregon, USA", "assisted suicide requests", etc. The nearest associati- on can be found in the term "Internet-related suicide" - but to be related to someone or something instead of being assisted by are two different things. Instead of being focused exclusively on scientifically approved resources, one also needs to look for less official and more popular definitions. 
In that regard, Wikipedia additionally offers an alternative definition of the term. It revolves around the identity-split between a "cyber/online persona" in the virtual social environment and a "real/physical persona" in the real social environment. It introduces a clear distinction between the two types of Internet suicide: one related to the "deliberate destruction of one's own online identity" (virtual life) and another related to the deliberate destruction of one's own "real" identity (physical life). The former case can be easily related to the existence of web sites such as the so- called Web 2.0 Suicide Machine operated by a medialab in Rotterdam [http:// suicidemachine.org/]. What can they do for you?

Basically, they offer "the social-media death", the possibility of self-erasure within your virtual social environment, "a service to users who want to drop out of their social-network." By using the logic of computer graphic design tools, they allow you to delete your own social networking profiles (on Facebook, Myspace, Twitter and LinkedIn), and also "to kill your fake virtual friends, thus completely doing away with your Web 2.0 alterego." A further and more radical reference is given by a digital media researcher Geoff Cox in his most recent text Virtual Suicide as decisive political act (Cox 2012) where, through the critical prism of communicative capitalism, he demonstrates "how the control of networked relations is increasingly managed through the dynamic interactions of technologies and biologies (the mixed reality management of life and death), the significance [of which] is explained in terms of the construction of certain kinds of subjectivity through the use of networked technologies". Among numerous other examples, he also describes Seppukoo (2009) in the following way: [...] a platform for users to commit virtual suicide in a ritualistic removal of their virtual identity. Making their conceptual references clear, the project title is an explicit reference to the Japanese ritual suicide of Seppuku (literally stomach-cutting) and evokes the stubborn refusal to fall into the hands of the enemy - and the preference for autonomy even at the cost of one's life. In such cases, suicide follows the Samurai code of honour (out of respect to the emperor, or perhaps disrespect to the empire in this case). Furthermore, the project is inspired by Seppuku!, the ritual suicide that some members of the Luther Blissett Project committed in 1999, to declare the end of their multiple identities project and the death of net.art as a temporary autonomous zone (Cox, 2012).

This is but one version of what we could understand by "cyber-suicide", which is a version of the term I would prefer to keep between the inverted commas. Another aspect is what I am mainly interested in here, the one in which one's own life could be finalized for real with the assistance of computer technology (due to the human involvement behind). However, Wikipedia - in its simple definition of the term (cybersuicide) - excludes any account on behalf of individual suicide cases, while highlighting the meaning of the cybersuicide (as opposed to the erasure of one's own virtual alter-ego) in relation to the group suicide ("such as suicide committed by several people simultaneously after meeting on the Internet"). On the contrary, when addressing the notion of "Internet suicide" or "net suicides" the definition does not 
exclude individual cases, but frames the performativity of the act in a very interesting way - in its relation to public visibility ("a suicide conducted in full view of the public via Internet") resulting from individuals' connectedness through a mutual online contact ("or pursuant to a cybersuicide pact, which is a suicide pact made between individuals who meet on the Internet").

Before engaging with the dimension of public visibility in online suicide rituals, I would like to reflect on the concept of (cyber) suicide pact. According to the recent research results (including: Becker and Schmidt, 2004, Rajagopal, 2004, Ozawade Silva, 2008, Naito, 2007, Westerlund and Wasserman, 2009) the definition of a suicide pact is summarized in Internet Pathways in Suicidality: A Review of the Evidence (Durkee et al., 2011) as following: [...] A cooperative choice by two or more individuals who agree that both, or all, will commit suicide together, in a prearranged place and at a designated time. Research concerning the Internet and suicidal behaviour, in this aspect, investigates what is known as "net suicides", i.e., suicide pacts made on the Internet. Evidence shows that suicide pacts often develop in chat rooms or on message boards, which endorse suicidal behaviours. This milieu attracts vulnerable persons feeling socially excluded from society. The socially-isolated individual can communicate interactively and anonymously, thus, exposing him/ herself to impending prosuicidal incitement from one or more parties involved (my italics). This could potentially lead to the coordination of a suicide pact. An example of such a tragedy occurred in Japan during 2004 when nine people took their own lives, in a suicide pact, initiated and coordinated over the Internet. According to Naito, in Japan alone some 60 persons a year are presumed to have died from 'net suicides' and these trends appear to be on the increase. However, this occurrence is not only limited to Japan. Online suicide pacts have been reported in other nations as well, including the United Kingdom, Norway and South Korea.

The curious case of organized group suicides over the Internet deserves a special attention and requires more space for elaboration than the format of this paper allows. However, it is important to stress a few elements in that context at this point: when examining this particular kind of suicide and some of its features as experienced in Japan, a social and cultural anthropologist Chikako Ozawa-de Silva locates the official origins of the phenomenon - the first reported case of Internet group suicide - in Korea, despite the prevailing view that tends to turn this epicenter back to Japan of the early 2000s (Westerlund and Wasserman, 2009; Durkee et al., 2011). Beside the precision of this kind of statistics, which could be arguable, I find the specificity of the Japanese context much more significant for another reason: the popular culture representations of Internet group suicide in the recent wave of Japanese movies and television series. These are the main sources that Ozawa-de Silva uses and analyses extensively and critically in comparison to the mainstream culture sources of representation as they are used and analyzed by the scientific public health institutions and their respective policy making bodies. In her own account, it is exactly in the popular movie-representations "where the moral blame for the suicides 
is targeted more at society itself rather than the individuals who are the victims of suicide; [...] the depictions of the popular culture social commentaries seem closer to the experiences and feelings expressed by the individuals who frequent Internet suicide forums" (Ozawa-de Silva 2010). This is where the direction in interpreting the pathological nature and reasons of the group suicidal phenomenon in Japan (and why not elsewhere) - is being reversed. This reversal (from a "suicide nation" toward a "social murder" through individual/group suicides) occurs in Ozawa-de Silva's important argument around the inevitable interdependency between the suicidal individuals and the (suicidal) society to which they belong: In contrast to "official" explanations of the rise of suicide in Japan, which center around Japan's economic stagnation and individual cases of pathology and depression, they [i.e. the popular culture representations] suggest that the Japanese individuals who visit suicide websites [...] are not isolated pathological cases, but victims and signifiers of a deep malaise or "social pathology" that is afflicting Japanese society. The individual psychology of such victims, therefore, is seen as inseparable from a social psychology that is in need of change and healing (Ozawa-de Silva, 2010).

What is in need of change and healing is the society itself, not only the individuals (the victims of the social pathology). Turning the arrow of pathology from a singular self toward the social (group) self, and doing so by highlighting the role of cultural (cinematic/visual) representations of suicide, Ozawade Silva gives an important possible argument to address the main goal of this paper as well. If, by our "safe (European) distance" from the "specificity" of Japanese locality, we would try to understand the universal message her argument has to offer, we could also dare to try and understand the "suicidal specificity" of our own (European) locality precisely by the current pathology of European society. Under the globalization regime there is, however, no place for such a long-distance disconnectedness between the two localities that seem to be geographically very far from each other. Even if they are very different indeed, it is the similarities between them that must be drawn. I would not be surprised if, sooner or later, those similarities turn to be so big that, at some future point, we could relate to Ozawa-de Silva's argument exactly by recognizing ourselves (as "Europeans") in what she indicates here: [...] The parallels between popular culture representations and suicidal behavior in Japanese society, with particular attention to the re-cent films Tokyo Sonata and Departures [...] do illustrate very well particular aspects of Internet group suicide that have captured the imagination of Japanese social commentators. This is especially important, because these are aspects that have largely been neglected in the approaches taken by the media and the government, but which, I will argue, are central to an understanding of Internet group suicide in Japan. (Ozawa-de Silva, 2010, my italics) The official approaches taken by the media and the government toward suicide at large need to be put under question, examined and balanced from other, complementary and "unofficial" perspectives. This does not only stem from my personal "myopic" perception and "suspicious" comprehension of the dominant ways of reading and interpreting the suicidal phenomenon (which accordingly leads to the do- 
minant ways of applying such readings into the official proposals and regulation policies for suicide prevention). Although it might sound so, it is not from the position of critique that I believe we must approach the official statements in the (online) context of suicide treatment: it is rather from the position that opposes any unilateral, one-sided approach (be it exclusively medical or exclusively Eurocentric) and pleads for the inclusion of the other - perhaps less relevant, perhaps equally valid - scientific and non-scientific disciplines and methods, such as popular and visual culture in ge-neral and the world of images in particular. This could contribute to the official grammar of writing and to the official perspectives of looking at suicide in a more nuanced and hopefully more beneficial way. How could these "unofficial" methods be functional in that sense?

\section{2. "INTERNET-ASSISTED" SUICIDE: A SOCIALLY RESPONSIBLE (VISUAL) COMMUNICATION}

Adding the "unofficial" (popular, cultural, visual) dimension to the official suicide-oriented research may allow us to think about the multiplicity and ambiguity of the ways by which the computer technology actually exerts its powers of assistance in the suicidal practice over the Internet. In the particular context of "Internetassisted" suicide I would dare to pose the following questions: Firstly, what (or who) is actually controlling the assistance protocol and how does it change over the time period during which a negotiation process about the decision of one's own life termination is taking place online? This especially concerns the assistance (either constructive or destructive) exerted upon the potential victim by other online users who participate in the same virtual discussion forum/social network on the Internet. Another question would concern the repercussions following the final decision by potential victims to subject themselves to the assisting service facilitated by the group pressure control under examination. Secondly, what is the distinction between the two types of cybersuicide as regards the number of potential victims organized around the Internet, namely the one undertaken by an isolated individual and another by the group consisting of two or more people determined to commit such an act online and together? Thirdly, what is the role that the mutual visual communication with the Other produces? Here I refer to the online communication between a potential victim and other members of the same discussion channel where the information exchange and the decision making are taking place (these can be either anonymous or familiar online interlocutors, whose invisibility or visibility depends on the usage of the visual tools such as the Webcam, accompanied by the audio tools such as microphone). I am here specifically interested in the ways by which the visual language (or the "mirroring effect" in the face of the Other over the computer screen) along with the written, spoken or heard language (such as exchange of messages via the usage of words or voice) has the potential to shape the attitudes of the individual either positively or negatively, thus provoking the assistance in suicide prevention but also - in suicide encouragement. 
These questions imply an ethical stance that I would like to address. This is an element by which the virtual, online, "real time" participation in the act of looking (at a self-destructive ritual via Webcam, for example) includes the level of responsibility that may or may not lead to drastic and tragic consequences on the other, "invisible" side of the computer screen; the other, "invisible" side of the screen, being the point from which the communication about the actual ritual of self-annihilation is produced (announced, planned, described, organized, staged, performed, made visible) is a decisive element in establishing the communication of an Internet-based ritual while (and this shall be highlighted) it is not limited only to the one-sided channeling of communicative suffering. On the contrary, it is articulated, produced and disseminated via the modes of dependence of a suffering individual upon the reactions coming from the other side of the virtual network of online users logged on to the same virtual public forum or interactive discussion format. Furthermore, it is enhanced by the visual modes of interdependence in the process of looking at each other. This includes all the repercussions, both positive and negative, that such an interdependence among the participants in the experience of opinion exchange (opinion making/fostering) through online networks implies.

The responsibility of virtual community becomes even more significant when it comes to a highly striking and prominent factor of ambivalence among the individuals in making the final decision (whether to commit, or not to commit, or to postpone the act of suicide). The factor of ambivalence can have beneficial effects (in terms of exerting a protective effect until support and treatment can be provided), whereas the responsibility of virtual community could be taken as exerting a positive influence (Westerlund and Wasserman, 2009). However, the factor of ambivalence can subject individuals to massive persuasion by other discussion participants that they are unable to back out or seek help for their problems which, in return, can produce in the suicidal "actor" the feeling of obligation [to other discussion participants] to proceed with the plans an individual had begun discussing and obtained reactions from other participants (Ibid.). The nature of such an encouraging influence of virtual community on the potential victim is further supported by the very principle of exclusion upon which the pro-suicide oriented websites operate, and on the victim's exposure to this one-sided encouragement to proceed with their suicide plans: The danger of websites of this type is thus what is more or less a chorus of voices in unison, inciting and urging individuals to commit suicidal acts, while voices with other solutions to life's problems are largely excluded; [...] since no opinions other than pro-suicide ones are tolerated at these websites their effect is to easily tip the ambivalent stance that most individuals with suicidal tendencies have regarding whether to go on living or end all (Becker and Schmidt, 2004, as in Westerlund and Wasserman, 2009).

Why does a suicidal individual need to establish contact with someone else, the channel of communication (online communication indeed) with the Other, in order to commit an act of self-destruction? Why the majority of the scientific analyses and 
reports on this issue tackle the problem almost exclusively from the verbal-centered methodology, embedded in the belief that it is only through the information exchange (written, spoken, heard; messages, notes, pieces of advice) that the online state of connectedness could impact one's own state of "disconnectedness" from the society? Is it possible to think beyond the verbal model of human contact as a dominant form of communication, in order to make it complementary with the non-linguistic methods of analysis? Could the visual nature of human communication and the visionexchange itself become treated as a language to be "learned' if we want to understand the phenomenon of cybersuicide?

The deeper we try to understand the scientific approaches to the phenomenon of cybersuicide based exclusively on the analysis of language (verbal communication as opposed to visual communication), the more it becomes evident that the institutional methods of investigation tend to treat Internet only as a tool (i.e. what is actually being used as a source of information). This is, of course, sanctioned given the fact that the Internet has become part of the broadly accessible repertoire of providers of suicide-oriented data, beside other, more traditional but less accessible sources, such as books, movies, or newsprint media - where the access to the relevant, practical in-formation on suicide methods, for example, is generally less accessible (due to the fact that the public media prohibit such materials in favor of those having the preventive nature). The role of the Internet and computer technology, on the other hand, should also be approached from other perspectives, and especially from those that treat new media not only as a tool but rather as a goal in itself (i.e. how it is used and why - apart from the individual's orientation toward information collection and verbal communi- cation exchange over the Internet). What do I mean by this?

Given its nature of a visual medium, let us approach the Internet as a medium without which the meaning of cybersuicide would not have had the same effect was it planned outside of the virtual social environment. This is all the more significant if we would accept the Internet as a visual medium with a global scope (in terms of its spatial outreach), and with an accelerated, immediate scope (in terms of its temporal outreach). Last but not least, the Internet is the form of communication that, by storing the textual and visual data online, gives an open access to this form of communication as timeless and omnipresent: "That is, everything can be stored and thus held onto in a lasting fashion. With the accumulation of a large body of information the fantasy of omniscience becomes possible" (Fiedler and Lindner, 2002). In such a way, it makes one's private suffering not only the matter of a temporary public concern, but also of the permanent public memory of one epoch - by documenting and archiving online the visual symptoms of the historical conditions in which suicide and cybersuicide take an active role.

In the given context of analysis, if I would attempt to define what I mean by "cybersuicide," I would first and foremost re-consider the fundamental shift occurring online by which we are also witnessing a new mode of "representation of suicide" in the virtual/cyber visual regime. This shift addresses the status of suicide 
under transformation within the time-space coordinates of the global and accelerated Internet- based visual regime. It could be defined in the manner referring to the transformation from an underexposed status of suicide toward an overexposed status of suicide. The "underexposure" denotes a subject that has always been strongly taboo and therefore 'belonged' to only a few voices in public discourse (Westerlund and Wasserman, 2009). The "overexposure" denotes a completely new situation where a taboo-subject is gaining the possibility of turning into a public discourse via the multiplicity of voices shared over the Internet, either with positive or negative reasons and consequences. This is where the radical discursive and visual turn is literally "happening" - by becoming not only a media event (conceived, produced and distributed via the medium of Internet) but also an interactive and visual media event (conceived, produced and distributed via the medium of Internet in order to become and remain hypervisible by mode of interaction with massive participants in the virtual social environment). In that sense, the relatively old presumption that "the Internet has a greater potential than other mass media to influence people in the direction of suicidal acts, mainly because of its interactive nature (Baume et al., 1997) becomes more than relevant once again, but from a radically different perspective. I am willing to understand the signi- ficance of interactivity in the media events of (live) cyber suicide spectacles as an essential element determining the reasons of conceiving, producing and distributing such spectacles online, and not uniquely because the public sphere of the Internet (as opposed to the public sphere of traditional media and institutions) allows access to sensitive/forbidden information regarding suicide, as in pro-suicide Web pages.

The exposure effect in itself reveals a particular turn - from the level of minimal offline visibility to the level of maximal online visibility. Therefore it testifies about the significance of the visual self-exposure and effectiveness experienced through the interactive media exchange on the Internet as an "iconic event" - an event embedded in the tradition of radical raptures in the history of the visual (and not only verbal, or word-oriented) cultural practices. No matter how cynical it may sound, for an anonymous and invisible multitude of global citizens the Internet provides an ultimate technological and visual possibility for the creation of an exclusive online identity that allows them, through virtual mirroring of the computer screen assisted by a Webcam, both to see themselves looking and to see themselves being looked at, simultaneously. If so far we have been oriented toward the "portrayal of suicide on the Web", it is time to turn toward the "suicidal self-portrayal over the Web" as a means of understanding the complexity of online media appearance and how it participates in the revealing of what is actually portrayed in the act of cybersuicide - the exposure of the suicidal subject itself.

Following the same line of thought, I find of an utmost importance the question posed by Westerlund and Wasserman around the manifestation of suicide event in Internet media (namely: "how is it presented there?"). This is a question that informs about the quantity and quality of online material contents about suicide (How 
much material about suicide is online and what is it about?). Nevertheless, I would dare to detach from the psychiatric concern with the modes of "presentation" of suicide e- vents and data as understood by experts in the medical scientific field (in terms of its quantitative and textual content-based data) in order to address the "representation" of the self (and consequently, the series of reenactments of a suicidal act through the rituals of multiplied online performances, generally recognized as copycat-suicides outside of the Internet context). My interest in the representation rather than presentation lies precisely in the visual nature of suicide - how it becomes visually communicated, engineered, performed, staged, recorded and documented over the Internet in the context of the live cybersuicide broadcasting, a phenomenon also known as "de-athcasting". In that sense, instead of exclusively relying upon the provision of a detailed discussion of suicide plans on suicide-oriented Web sites and forums, I would rather favor a definition of cybersuicide that, as " $a$ slang term used to describe a suicide or suicide attempt that has been influenced by Web sites on the Internet [denotes] a public showing of the suicide or suicide attempt when the victim uses a Webcam to record the suicide attempt" (Webopedia, 2012). I am here insisting on two particular aspects: on the popular usage of the term as part of a "deviated", slang version (instead of its mainstream linguistic format as proposed by scientific literature); and also on the usage of the Internet-related devices (such as visual screen and Webcam, for example) as evidence of someone's serious intentions and also as tools that could bring us closer to the "pictorial" (visual) aspects of the phenomenon of cybersuicide.

To sum up: under the term "cybersuicide" I understand the role that the Internet can play as a showcase for the public display of (self-inflicted) death, along with the mediatory assistance performed by the computer-based textual and especially visual tools (such as the screen and the Webcam), the biological visual tools (such as the eyes of a spectator, or the multiplicity of eyes behind the screen), and the social tool (the responsibility of other participants in online communication and their assistance in the potential victim's final decision-making). By posing a question "What is actually represented/showed/dis-played via the Internet through cybersuicide?" (at the moment when one's own decision about the self-inflicted death is already made and is being staged for the gaze of the other via the Internet), I am willing to argue that it is the "suicidal subject" itself (the object of our suicidal gaze) that we are witnessing in the live cybersuicide broadcasting as the public display of death: the suicidal gaze being that kind of gaze within the broader consideration of the history and theory of vision that has become inseparable from the new formats of assisted suicide practices at the turn of the millennium and especially in the first decade of the twenty-first century, namely the "Internet-assisted" suicide. The reason why I would insist on framing this term by quotation marks lies exactly in the necessity not to underestimate the responsibility of the humans behind their machines in influencing the decisions of the victims (cyber bulling, for example). As it is not the Internet that assists in the suicidal ideation and execution of the self, but the participants in online communication themselves (the onlookers, beholders, supporters, commentators). 
This would prove to be an additional contribution to the prevailing claim (yet always questioned anew) that the Western thought and culture have been dominated by a vision-centered para- digm of knowledge, ethics and power (Levin 1993) that we need to consider and re-consider each time we are supposed to reflect upon the hierarchies of visual regimes and optical machines within which our everyday life, willingly or not, resides. Because what is essentially shared over the Internet in the cybersuicide pacts is not (only) the information and communication about the suicide itself, but exactly the gaze (the suicidal gaze) as the consequence of the practice of looking: and a very special kind of looking, indeed - the one that is highly dependent on the logic of the human responsibility, lost in the mysteries of human relationships.

\section{FROM HYPERTEXTUAL SELF TO HYPERVISUAL SELF. A CASE OF MARCUS JANNES (2010)}

I would like to evoke a case that has been exemplary for my personal interest in the study of the relationship between the notion of suicide and its implications over the Internet. On October 11, 2010, a twenty-one year old Marcus Jannes killed himself in Stockholm. He hanged himself during a live broadcast on the Internet. Police described this relatively unknown phenomenon in Sweden as rare in that country. Preceding the act, he had described himself to an online community of virtual beholders, in his own words, as an emotionally vulnerable and lonely person who, on the top of it, suffered from a high-functioning autism (Asperger syndrome). A local webmedia posted a report the day after. The situation in which the lethal act had occurred was described under the headline "Swedish man dies in live 'cyber suicide' broadcast" (The Local, 2010).

This has not been a unique case in the history of suicidal events transmitted live over the Internet. Throughout the last decade there have been more tragic examples of this kind, such as those in India (2004 and 2008), Britain (2007), and Florida (2008). This excludes the numerous online suicide cases without any use of web-cameras, as well as those "offline" cases where the mobile camera devices (as in cell phones) were used without any Internet assistance. The curious case of Marcus Jannes was the first one I got provoked by, however. One of the reasons that triggered my attention the most was the online availability of the visual traces following his death: what was particularly troubling about it to me was not the available information about one's own self-execution over the Internet, but rather its character of an event that was first broadcast live and then archived online among the YouTube depository of video materials of the most various kinds.

The video of the Swedish live cybersuicide broadcast, no more available over YouTube to my knowledge at the moment of writing (Spring, 2012), confronts us with the image of the space where the "event" took place. The image was recorded by a web-camera positioned in the left corner of the room. What we actually see is the interior of a domestic ambiance (a neatly arranged room in Jannes' flat). Its back- 
ground shows a huge tapestry hanging on the purple-colored wall; a balcony-door is next to the internal passage leading to the rest of the house; the floor, covered by two pieces of white rug, separates the background from the foreground which is defined by green leaves of a plant (to the left) and the backside of a computer monitor or a flatscreen TV on the table (to the right). Two small boxes rest next to the screen. A striking detail is right in front of our eyes: a white rope leaning on the edge of the balcony door. For all the belated spectators of this "scenography" (being a posthumous digital "stage" of the cybersuicidal event), the lower right section of the image makes the impression about the video material even more disturbing. It contains the words ViralDeath.com. reality as it is. This is certainly true once the "action" starts taking place: we see a young man in black trousers and a red T-shirt (with a popular fashion company slogan Just do it!). He is walking nervously around the space before putting a noose around his neck. The quick set of "actions" leads to a tragic end: he takes some liquid, slides down onto the floor, the rope is pulled and he hangs himself. The second available YouTube video shows the aftermath of the "event": a couple of police officers enter the room and investigate the body before its final removal. They are themselves recorded by the same web-camera as it was continually running without control once the suicide was committed.

One hundred years ago this would have been an unimaginable event simply because the global communication network has not yet existed, while the phone/radio communication did not function to such an extent; technological and media tools allowing the live communication (simultaneously visual and verbal) across the world were lacking; there was no Internet or Web-camera that could transmit images, sounds and words to innumerable audience scattered around the world at the same time while the central event was taking place in an intimate, private environment of one's own flat. Nevertheless, one simple optical tool was available indeed. An infamous case that took place in Vienna in 1908 (more than a century before Marcus Jannes decided to leave this world forever) still triggers my attention: not for being yet another self-destructive ritual happening in the general cultural and psychological climate colored by deep, even spiritual introspection in the finde-siècle Austro-Hungarian Empire, but precisely due to the logic upon which that self-destructive ritual relied on the available optical tool itself. The facts that the victim was one of the most promising young painters in early twentieth-century Vienna, that he never exhibited, and was barely known in his lifetime, all make little importance for this paper. But the fact that - precisely thanks to his visual mastery and sensitivity toward the laws of vision and the power of looking - he understood the dark side of the logic of the human gaze, makes his case complementary to Jannes' live cybersuicide broadca- sting and deserves to be mentioned in the same context. Close to the family circle of famous Austrian composer Arnold Schoenberg (and certainly too close to his wife), Richard Gerstl precedes Marcus Jannes in the sense that he committed suicide following the similar logic of the gaze, yet in its "primitive" form, in comparison to the early twenty-first century. What did Gerstl do at the moment of his nervous breakdown? He piled his paintings underneath him in his studio and, in an 
act that must have been as acrobatic as it was brutal, simultaneously plunged a knife into his heart and hanged himself over the burning canvases (Jones, 1994). But this is not the most interesting part, no matter how horrifying it may sound. Before tightening a noose around his neck, Gerstl positioned himself in front of his atelier mirror, and plunged a butcher knife into his chest (Harrison, 1996).

Gerstl's suicidal self-portrayal remains executed in the isolating fashion of a solitary and silent act, a personal perceptual narrative divisible between his physical and his "virtual" self. His own image, duplicated on the glass surface of the mirror, remains narcissistic and entirely self-centered inasmuch as it intentionally avoids any exchange with the gaze of the Other and remains "offline" - disconnected from the eye of the society. However, it is not the image but the vision, the way of looking, that becomes a fascinating issue in his case. Gerstl's visual experience of the reflected self in the mirror was one way of establishing a direct contact with the ultimately invisible and subjective realm of death, "the other side" of life being the site beyond all things visible, behind the surface of the mirror. By looking at himself dying, by seeing his own death through the act of self-annihilation, he acknowledges - to his own gaze (his own lethal "point of view") - the true power of the nature of human contact with the replica of his/her own image. His own gaze, which acts as his observing ego, becomes therefore the subject (not the object) of looking positioned exactly in the image-field of the mirror. Gerstl himself, being therefore the object (not the subject) of looking, sacrifices himself to his own self-aware image projected in the mirror; this projected image, being self-aware inasmuch as it possesses the power of control (of looking), functions precisely in the way that returns the projected gaze back to the beholder thus making the execution (of the exposed physical self) possible. The "suicidal gaze" of the image destroys the gaze of the image-maker. For Freud, this would be an exemplary case: "Probably no one finds the mental energy to kill himself unless, in the first place, in doing so he is at the same time killing an object with whom he has identified himself and, in the second place, is turning against himself a death wish which had been directed against someone else" (Freud, 1974; Anderson and Plaut ,1999, my italics). Who might have been "someone else", apart from his own image reflected in the mirror, against whom a twenty-five year old Gerstl directed his death wish in the Gesel-schaftsspiegel (mirror of society)? Curiously enough, it was exactly in a small German socialist journal Geselschaftsspiegel that Karl Marx's only published discussion of suicide appeared in 1846. Composed during his short living period in Belgium, having been expelled from France for his political activities (Anderson and Plaut 1999), it gives but one yet arbitrary association with the intricate relationship between early modernist suicides and their "mirroring effect".

In his own way of using images (and particularly the image of himself), Gerstl left a statement about himself and his place in the concrete historical situation of the early twentieth century. I would dare to ask questions now that might sound as absurd as arbitrary. This equally holds true if those questions could have been posed 
by any of Gerstl's contemporaries - which would be an impossible perspective for the people of 1908. What I am curious about is the following: were Gerstl the man of today, a contemporary of Marcus Jannes, what could he have done instead of looking at himself in the mirror? What would the paradoxical simultaneous operation look like if he had a Webcam instead of a mirror? By the "paradoxical simultaneous operation" I mean: the reproduction of the image of oneself (as reflected upon the mirror surface) existing simultaneously with the destruction of the image of oneself (as a consequence of the suicidal act). How to bring together two images, or two types of self-centered vision, that are supposed to emerge and exist together for a very short period of time if for the one, in order to exist, the other must be necessarily and immediately excluded from the sight? How to reconcile the two impossible perspectives situated in the conflict of self-observation: the situation in which the duplication of one's own gaze in the mirror coincides with the annulation of one's own gaze in front of that mirror?

These are the questions to be posed again in the future. It might be that the answers have already been given long ago: but for that part of mankind, whose certainty of being lies only in the privileged domain of the verbal, those answers may never be clear as they might not be easily translatable into the speech system. Would it be enough to look in order to understand? Would it make more sense to look at the series of images that Schoenberg, a composer taught to paint by younger Gerstl, produced in the aftermath of Gerstl's death and simply named them "The Gaze"? The potential parallels have already been drawn: according to his student and friend Victor Hammer, Gerstl "placed great importance on the 'expression' of the eyes", while the relationship of Schoenberg's "eyes" to similar expressive gazes in portraits and particularly self-portraits by Richard Gerstl, whose work predates Schoenberg's paintings, is striking. (Brand and Hailey 1997).

For this moment, let us go back to Gerstl-as-Jannes. What could he have done instead of what he actually did were he the man of our times? He might have connected himself online in order to stage a live broadcasting of his "performance"; he might have tried to set up his Web camera and log himself on a popular Internet forum; accordingly, he might have activated his virtual social network in order to present himself by his full name and his age, to provide a description of his social conditions and living status, to write down his messages (first the warning ones, then his final note later on), to create the atmosphere of an event (no matter how unusual) and to stage his appearance and communication with other online participants (in a so-called suicide pact, or without it); he could have also provided an FTP link (with login information for the others to access the photos of the event that he had meanwhile recorded) where he would reveal his intentions and reasons to die. Finally, instead of killing himself in front of the mirror, he could have performed his act in front of the virtual community of online observers - which is what Marcus Jannes actually all did, one hundred years later. What would have remained after such an event? A digital video-trace: a moving image available online, for the time after, 
when the innumerable Internet audience curious to watch his last action would have a possibility to access the spectacle freely for the viewing, at their own pace, whenever they would want, and as many times as they would want.

The suicide in Europe has taken on a new meaning again: it has become an unbridled pursuit of a global visibility, the transnational character of which resides exactly in the possibility offered by the role played by images in the new media system of message-distribution. Here there is an effect that goes in line with (but also beyond) all intentionality of the victim: it is not only being recorded and transmitted in real-time, but also archived online and repeatable over the Internet for a massive consumption at any given moment in time by the multitude of anonymous onlookers. For the man thus exposed, is it assistance (in the process of looking) that becomes a mode of survival, of overcoming death, a "liberation" from it - as opposed to nonassistance which leads to the condemnation of death? We cannot speak on behalf of those who were behind their computer screens that October day, experiencing the live broadcast of Marcus Jannes' suicide event in real-time (here I am thinking both about the experience of the victim and the experience of his virtual witnesses). Our lack of personal experience, I believe, denies the possibility of having any openly judgmental and critical or, in the worst case, even affirmative stance about what, how, and why (and finally for whom) it happened. However, what we could do is to focus on the textual and visual traces - the fragments of the event - as they were recorded, described or remembered (and eventually interpreted) by the online media such as news portals and YouTube. It is via those mediated (posthumous) traces of information that we can try and ask ourselves: what Marcus Jannes' case means, if anything, for each and every one of us and for the times we live in? What does a live broadcast of one's own self-destruction on the Internet actually show us? What kind of "invention" in an adventure of looking? Does it embed itself in the tyranny of images or in the pathology of images, indeed? Or something else? More generally speaking, how does the intentional act of self-destruction equates with the information technology, computer- networks and the Internet? And what are the implications of such a correlation to our everyday life, i.e. not only in relation to the potential or actual victims of self-destruction, but also to the potential or actual witnesses, accomplices, participants or "survivors" in the ritualization of self-destructive events on the Internet?

\section{CONCLUSION}

To respond to the question "What should be done?" is not easy. One answer could be given by the recommendations issued by experts in the field of psychiatry that are important and necessary for numerous reasons (and here I will rely on the selection of those proposed by Westerlund and Wasserman, 2009): (1) they testify how globalization and the emergence of new communications technology also create new challenges to global health; (2) they urge the performance of more studies, with 
more precise measuring methods, to allow us to see whether Internet-based communication affects the incidence of suicide and, if so, how; (3) they advise Internet companies providing search engines, through optimization strategies, to attempt to maximize the probability of suicidal individuals finding websites that offer help and support instead of the risk of being ushered onto websites that are dangerous to life; (4) they indicate the importance of psychiatric stuff asking patients about their use of the Internet, and also helping them find websites with preventive content and supportive functions, so that the Internet user can exert a therapeutic effect instead of constituting a threat. But, beside all the efforts to capture the power of the Internet regime in exerting the modes of social control over suicide-oriented Web users, one thing nonethe- less remains: "[...] although Internet-based communication is extremely widespread today, know-ledge of how it affects suicidal behavior is virtually non-existent" (Westerlund and Wasserman, 2009, my italic).

If we are going to start almost from the scratch (that scratch being present for more than fifteen years now in scientific literature and laboratories at least in the socalled developed world (see Holland, 1995 and Young, 1996), we need to build up our mind with knowledge, and general knowledge indeed, not a narrow-focused perspective on the so-called cybersuicide. This also implies the need to update our understanding of contemporary media events within the analysis of globalized media cultures, and to raise the question of how the visual online communication ("the images that mobilize conscience", in Susan Sontag's terms, that are "always linked to a given historical situation") affects the suicidal behavior today. I consider this issue to be pressing even more so because the knowledge of how images are affecting our e-veryday life, and how they have been affecting the history of our civilization, has been developed throughout the centuries behind - and is still under development: it could hopefully serve as a useful tool in coping with the atrocities of our own epoch. These might be the atrocities caused by our own hand under control by our own social, economic, political and technological realities in the given historical situation. And our own epoch might be that long techno-capitalist era colored by the mankind whose "self-alienation," as Benjamin wrote in 1936, "has reached such a degree that it can experience its own destruction as an aesthetic pleasure of the first order".

To my knowledge so far, few of the critical accounts of the direct relationship between suicide and the Internet have touched upon the role that the screen (and the image/digital image/video transmitted via the computer screen) has played in the pro-cess. Curiously enough, the criticism focused directly on this relationship has had more to do with the somewhat bizarre cases such as the internet image of a religious cult The Heaven's Gate mass suicide, or the video image on the television screen of a "real-life" killing executed by the American doctor Jack Kevorkian ("Dr Death") imprisoned for carrying out an assisted suicide (Brown 2001). In the last few years online content has grown with amateur video materials showing the instances of self-inflicted death for various reasons and from across the world. The eighteenth-century notion that images carry extremely powerful messages and can 
be influential in cau- sing suicide (Knox 1778, Brown 2001) does not prevent us from questioning their influence and meanings they generate in our own times. On the contrary, it urges us to approach them in an as extended methodological way as possible, so that the troubling and all the more increasing relationship between suicide, the Internet, and all the protagonists in the online suicidal rituals, could be approached in a more comprehensive manner for the sake of understanding and, consequently, preventing such a phe- nomenon to occur more often.

\section{BIBLIOGRAPHY}

Alao, A.O., Soderberg, M., Pohl, E.L., Alao, A.L. (2006), „Cybersuicide: Review of the role of the Internet on suicide“, Cyberpsychol. Behav. Vol. 9, pp. 489-493.

Baume, P., Cantor, C.H., Rolfe, A. (1997), „Cybersuicide: the role of interactive suicide notes on the Internet", Crisis, Vol. 18, pp. 73-79.

Becker, K., Schmidt, M.H. (2004), „Internet chat rooms and suicide. J. Am. Acad. Child Adolesc", Psychiatry, Vol. 43, pp. 246-247.

Benjamin, W. (1936), The Work of Art in the Age of Mechanical Reproduction. http://www.marxists.org/reference/subject/philosophy/ works/ge/benjamin.htm

Birbal, R., Maharajh, H.D., Clapperton, M., Jarvis, J., Ragoonath, A., Uppalapati, K. (2009), „Cybersuicide and the adolescent population: challenges of the future?“, Int. J. Adolesc" Med. Health., Vol. 21, pp. 151-159.

Brand, J., Hailey, C. Eds. (1997), Constructive Dissonance. Arnold Schoenberg and the Transformations of Twentieth-Century Culture, The University of California Press, Berkeley

Brown, R. M. (2001), The Art of Suicide, Reaktion Books, London.

Coffer, R. (2011), Richard Gerstl (1883-1908), http://www.richardgerstl.com/

Cox, G. 2012"Virtual suicide as decisive political act" in W Sützl \& T Hug (eds), Activist Media and Biopolitics: Critical Media Interventions in the Age of Biopower, University of Innsbruck Press, Innsbruck, pp. 105-118.

Durkee, T., Hadlaczky, G., Westerlund, M., Carli, V. (2011), „Internet Pathways in Suicidality: A Review of the Evidence“, Int. J. Environ. Res. Public Health, Vol. 8, pp. 3938-3952.

D'Hulster, N., Heeringen, C. (2006), The Role of the Internet in Suicidal Behavior (Cyber- suïcide. De rol van het internet bij suïcidaal gedraag). Tijdschrift voor psychiatrie, Vol. 48, No. 10, pp. 803-807

Ferranto, M. (2010), "Digital Self-Fashioning in Cyberspace: The New Digital SelfPortrait" in A History of Visual Culture. Western Civilization from the 18th to the 21st Century, J. Kromm \& S. Benforado Bakenwell (Eds.), Berg Publishers Oxford \& New York, pp. 356-366.

Fiedler, G., Lindner, R. Suicide Fora on the Internet (Suizidforen im Internet) (2002), Suizidprophylaxe, Vol. 29, pp. 26-31. http://www.magpiebridge.net /suicidefora.htm

Flashback forum (2010), https://www.flashback.org/t1322408 
Freud, S. (1974), The Standard Edition of the Complete Psychological Works of Sigmund Freud, Vol. 24, Strachey, J. (trans.), Hogarth Press, London.

Harrison, T. (1996), 1910: The Emancipation of Dissonance, The University of California Press, Berkeley

Holland, N.N. (1995), The Internet Regression, www.rider.edu/u-sers/suler/ psycyber/holland.html

Internet World Stats, Updated 8 April 2012, Retrieved 2012-04-15, http://www. internetworldstats.com/

Jones, M.E. (1994), Dionysos Rising: The Birth of Cultural Revolution Out of the Spirit of Music, Ignatius Press, San Francisco.

Knox, V. (1778), Essays Moral and Literary, vol.1, London, pp. 247-251.

Levin, D.M. (1993), Modernity and the Hegemony of Vision, University of California Press, Berkeley.

Naito, A. (2007), „Internet suicide in Japan: Implications for child and adolescent mental health", Clin. Child Psychol. Psychiatry, Vol. 12, pp. 583-597.

Nivtova, A. (2010), Swedish Teenager Hangs Himself in Live "Cyber Suicide", http://www.globalcrisisnews.com/europe/swedish-teenager-hangs-himself-inlive-cyber-suicide/id=1872/

Ozawa-de Silva, C. Shared Death (2010), „Self, Sociality and Internet Group Suicide in Japan“, Transcultural Psychiatry, Vol. 47, pp. 392-418. http://www. anthropology.emory.edu/FACULTY/Ozawa/TP-09-0013.R3-3-18-10FINAL.pdf

Ozawa-de Silva, C. (2008), „,Too lonely to die alone: Internet suicide pacts and existential suffering in Japan“, Cult. Med. Psychiatry, Vol. 32, pp. 516-551, http://www17.ocn.ne.jp/ ogigaya/keio/CMPOzawadesilva2008.pdf

Rajagopal, S. (2004), „Suicide pacts and the Internet“, BMJ 2004, Vol. 329 (7478), pp. 1298-1299.

Plaut, E.A., Anderson, K. Eds. and Transl. (1999),, Marx on Suicide, Northwestern University Press, Evanston, Illinois.

Sher, L., Vilens, A. Eds. (2009), Internet and suicide: An overview of the first decade of research, ideas and debates, Nova Science Publishers. New York.

The Local (2010), Swedish man dies in live 'cyber suicide' broadcast. http://www.thelocal.se/29566/20101012/

Wakefield, M.A., Rice, C.J. (2008), The impact of cyber-communication on today's youth, ACAPCD, Vol. 14, Alexandria, American Counseling Association, VA, http://counselingoutfitters.com/vistas/ACAPCD/ACA-PCD-14.pdf

Web 2.0 Suicide Machine (2009), http://suicidemachine.org

Webopedia (2012), http://www.webopedia.com/TERM/C/cyber-suicide.html

Westerlund, M., Wasserman, D. (2009), "The Role of the Internet in Suicide Prevention" in Oxford Textbook of Suicidology and Suicide Prevention. A Global Per-spective, D. Wasserman, C. Wasserman (Eds.), Oxford University Press, Oxford, pp. 525-532. 
Wikipedia contributors (2012), "Cybersuicide", Wiktionary, The Free Multilingual Dictionary, http://en.wiktionary.org/wiki/cybersuicide

Wikipedia contributors (2012), "Internet suicide," Wikipedia, The Free Enciclopedia, http://en.wikipedia.org/wiki/Internet_suicide

Young, R.M. (1996), Primitive Processes on the Internet, http:// human-nature. com/rmyoung/papers/prim.html 